\title{
Engineered Retroviruses as Fluorescent Biological Reference Particles for Small Particle Flow Cytometry
}

\author{
Vera A. Tang ${ }^{1,2,}$, Anna K. Fritzsche ${ }^{1,2}$, Tyler M. Renner ${ }^{2}$, Dylan Burger ${ }^{3}$, Edwin van der Pol ${ }^{4,5,6}$, \\ Joanne A. Lannigan ${ }^{7}$, George C. Brittain ${ }^{8}$, Joshua A. Welsh', Jennifer C. Jones, \\ and Marc-André Langlois ${ }^{1,2,10, *}$
}

\begin{abstract}
There has been renewed interest in the use of flow cytometry for single particle phenotypic analysis of particles in the nanometer size-range such as viruses, organelles, bacteria and extracellular vesicles (EVs). However, many of these particles are smaller than $200 \mathrm{~nm}$ in diameter, which places them at the limit of detection for many commercial flow cytometers. The use of reference particles of diameter, fluorescence, and light-scattering properties akin to those of the small biological particles being studied is therefore imperative for accurate and reproducible data acquisition and reporting across different instruments and analytical technologies. We show here that an engineered murine leukemia virus (MLV) can act as a fluorescence reference particle for other small particles such as retroviruses and EVs. More specifically, we show that engineered MLV is a highly monodisperse enveloped particle that can act as a surrogate to demonstrate the various effects of antibody labeling on the physical properties of small biological particles in a similar diameter range.
\end{abstract}

KEY TERMS: Small particle flow cytometry (FCM), nanoscale flow cytometry (NFC), retroviruses, biological reference particles, extracellular vesicles (EVs), exosomes.

Flow cytometry (FCM) analysis of viruses less than $500 \mathrm{~nm}$ in diameter has been reported since the late 70 's (1-3). Much of this foundational work required customized cytometer configurations, including high powered lasers, large collection angles, and very low sampling rates. Advances in the technology of modern cytometers now allow for some conventional commercial instruments to detect biological particles down to the $100 \mathrm{~nm}$ diameter range with minor to no modifications to default instrument configurations (4-14). However, several key challenges remain for small particle FCM and these include: variations in instrument configurations and detection capabilities across platforms and facilities, widely differing sample processing and labeling methods, and a lack of consensus for data reporting (15).

One of the major factors impeding these efforts for standardization is the paucity of available reference particles with fluorescence intensities relevant to that of biological samples (16). Reference particles are important for daily quality control of instrument performance, as well as internal positive controls for 
Tang et al., 2019

optimization of sample labeling protocols. Particles with low levels of fluorescence are needed to ensure optimal signal to noise resolution for dim signals. Biological reference particles have the advantage of possessing similar biochemical composition and antigen density levels, and can therefore act as suitable positive staining controls for antibody and dye labeling assays.

The development of calibration particles is also critical for the standardization of reference particle and sample data analysis and reporting in standard units. Although fluorescent reference particles in the form of polystyrene beads are commonly available, these are several microns in diameter and generally do not exhibit comparable fluorescence intensities (i.e., they are too bright) as EVs or even viruses. The calibration of fluorescence axes for small particle analysis using molecules of equivalent soluble fluorophore (MESF) units will require a set of populations that are smaller and dimmer than those currently available. Light scatter calibration using Mie modeling on the other hand requires homogeneous, well-characterized particles, with a variety of diameters and refractive indices (RIs) that are not necessarily the same as biological particles. Currently there are few sources of wellcharacterized light scatter reference materials for small particle FCM. Calibration particles and reference particles are therefore two distinct groups of materials that fulfill different roles with small particle standardization; the development of both, however, is required in the pursuit of standardized small particle FCM assays.

The murine leukemia virus (MLV) is symmetric and roughly spherical in shape, with a diameter of $124 \pm 14 \mathrm{~nm}$ as measured by electron cryomicroscopy (17). The specific strain used in this current study, Moloney MLV, is an ecotropic murine gammaretrovirus, meaning that it can only infect certain strains of susceptible mice(18). The viral envelope is primarily derived from the plasma membrane of infected cells, acquired during viral egress; a process that shares several common pathways with EV release into the extracellular medium $(19,20)$. The precise and consistent stoichiometry involved in virion capsid assembly results in the release of particles that are monodisperse in structure. This is a critical and highly desirable feature, which distinguishes viruses from other biological reference particles. MLV naturally expresses on its surface host cell-derived markers, along with the viral envelope glycoprotein (Env). Env is expressed as a trimeric structure with a transmembrane domain (TM) and a surface (SU) antibody-accessible subunit $(21,22)$. For most retroviruses, Env constitutes the only viral protein expressed on their surface. The number of Env trimeric structures, termed spikes, is a feature that has been characterized for several retroviral species. For example, while the human immunodeficiency virus type I (HIV-1) expresses approximately 14 - 21 spikes per particle, the simian immunodeficiency virus (SIV) was shown to have 73-98, the Rous sarcoma virus (RSV) 82, and MLV 100 (23-26).

For this study, we engineered a fluorescent MLV expressing superfolder GFP (sfGFP) as a fusion protein with Env $(8,27)$. The fluorescence of EnvsfGFP was quantified using MESF beads $(28,29)$. The unique features of viral homogeneity for both diameter and Env-sfGFP expression levels enabled the use of MLVsfGFP as a prototypic small vesicular particle to demonstrate quantification of fluorescence expression as a means to enumerate viral surface protein expression, as well as address pertinent questions regarding antibody labeling of small particles using Env-sfGFP as the target antigen. These include: 1) the relationship of fluorophore diameter and brightness to the resolution of small particle populations, 2) the impact of antibody labeling on diameter and RI, and 3) whether the use of multiple antibodies can impede optimal labeling and fluorescence intensities.

The use of this strain of MLV as a reference particle poses no biosafety concerns since they are ecotropic mouse viruses that are readily inactivated with formalin. They can also be lyophilized for stable storage and transport. The ability for them to be engineered to express surface epitopes of choice, fluorescent or otherwise, make these ideal controls for $\mathrm{EV}$ and virus immunophenotyping experiments. Based on these characteristics, we conclude that MLV particles exhibit essential features of a biological reference particle, and provide a much- 
Tang et al., 2019

needed tool for daily quality control, positive controls for select protein markers, and a simple method for evaluating cytometer sensitivity.

\section{MATERIALS AND METHODS}

MLV production. Generation of chronically infected NIH 3T3 cells and the production and preparation of MLV samples for flow cytometry analyses were described previously (8). MLVsfGFP was engineered from a glycogag-deficient MLV using overlapping primers to insert the sfGFP sequence into the proline-rich region of Env using a restriction-free cloning strategy, hence allowing for its surface expression as a chimera with the viral protein (27). Viruses were harvested from the supernatant of chronically infected NIH 3T3 mouse fibroblasts and directly analyzed by FCM. Briefly, for virus production, $2.5 \times 10^{6}$ chronically infected cells were seeded into a $10-\mathrm{cm}$ dish and cultured for 12 hrs. Cells were then washed to remove the serumcontaining media and further cultured for $72 \mathrm{hrs}$ in $10 \mathrm{ml}$ of phenol red-free DMEM (WISENT Inc.) supplemented with $10 \%(v / v)$ EV-depleted fetal bovine serum. The cell supernatant was collected and passed through a $0.45 \mu \mathrm{m}$ filter. The supernatant was then diluted with $0.1 \mu \mathrm{m}$-filtered PBS (WISENT Inc.) as required for analysis.

Quantification of MLV particle concentration and coincidence detection. Viruses are produced at a constant rate by chronically infected cell lines. The concentration of virus in the supernatant from infected cells correlates directly with the number of infected cells seeded (Suppl. Fig. 1A). Particle concentration of viruses was determined based on virus-gated events using 1:1000 dilution, which usually yields a concentration of particles $\sim 1-5 \times 10^{6}$ particles/ml as determined by the Beckman Coulter CytoFLEX S with a sampling rate of $10 \mu \mathrm{l} / \mathrm{min}$. Volumetric counts obtained from the CytoFLEX were validated by NTA (Suppl. Fig. 1B and 1C). Serial dilutions of the MLV containing supernatants show consistent SSC and fluorescence intensities at dilutions below 1:500, with $\leq 1 \%$ electronic abort rate (Suppl. Fig. 1D to 1F).

Flow cytometer set-up, beads, and data acquisition. Unless otherwise indicated, all samples were acquired on a Beckman Coulter CytoFLEX S with 4 lasers (405 nm, $488 \mathrm{~nm}, 561 \mathrm{~nm}, 640 \mathrm{~nm})$, using $405 \mathrm{~nm}$ SSC-H (405-SSC-H) as the threshold parameter (threshold at 1400 a.u.). Detector gain for fluorescence and SSC detection were optimized using MLVsfGFP, with $0.1 \mu \mathrm{m}$-filtered PBS used as the background control for threshold determination. The gains for the respective detectors associated with the following spectral filters: 405-SSC, 405-450/50, $488-525 / 40,561-580 / 30$, and $640-670 / 30$ were 1400 , $1200,3000,1600$, and 1200 a.u. respectively. A 405SSC vs. time plot was used during acquisition to monitor, and ensure, consistency of the event rates. All samples were acquired for $1 \mathrm{~min}$ at a sampling rate of $10 \mu \mathrm{l} / \mathrm{min}$. The sampling volume was validated by weight using the CytExpert volumetric calibration tool. The CytoFLEX Sizing Mix (prototype) (Beckman Coulter, Brea, CA) was analyzed undiluted and ApogeeMix (Apogee Flow Systems, Hemel Hempstead, UK) was diluted 1:5 with $0.1 \mu \mathrm{m}$-filtered PBS for analysis. FlowJo v.10 (FlowJo, LLC, Ashland, OR) was used for analysis of flow cytometry data.

Imaging Flow Cytometry (IFC). All MLV samples were acquired on a two camera ImageStreamX MKII (LuminexCorp.) according to the method previously described (12), with the modification of using the $405 \mathrm{~nm}$ laser $(120 \mathrm{~mW})$ for scatter measurements. Briefly, samples were acquired with $60 \mathrm{X}$ magnification, eGFP excitation with a $200 \mathrm{~mW} 488$ $\mathrm{nm}$ laser, and scatter with the $405 \mathrm{~nm}$ laser described above. Emissions were collected for scatter in $\mathrm{CH} 07$ (bandpass 405-505nm) and $\mathrm{CH} 02$ for eGFP (bandpass 480-560 nm). All samples were acquired using the Inspire software and collected for a period of two minutes using a scatter acquisition gate that eliminated the speed beads $(1 \mu \mathrm{m}$ polystyrene beads used for camera synchronization). Instrument sheath and sample dilution buffer was a $0.1 \mu \mathrm{m}$ sterile filtered DPBS/Modified (HyClone cat. \#SH30028.02). Buffer only controls were also run for the same amount of time to be sure that the same volumes were acquired as the samples. All virus samples were run in triplicate. $500 \mathrm{~nm} \mathrm{Si} 7$ peak FITC-MESF beads were also acquired using the same instrument settings as the virus samples. Data was processed using IDEAS 6.2 software (LuminexCorp) and FCS data files created for the scatter and GFP parameters and submitted for further analysis by the 
Tang et al., 2019

University of Ottawa Flow Cytometry and Virometry Core Facility.

Fluorescence standardization and quantification using MESF beads. Calibration curves were generated using a linear fit by plotting the known MESF values vs. their respective fluorescence intensities in linear scale for each of the MESF bead sets used in these studies. The beads used were 500nm Si FITC-MESF (30), BD QuantiBrite PE (Lot 73318, BD Biosciences, Mississauga, ON), and Quantum-5 FITC MESF Beads (Bang Laboratories, Fishers, IN). Autofluorescence was measured using the blank bead population, and this was subtracted from the fluorescent-bead values. The uncertainties of the fluorescence values for each bead population was accounted for in the generation of the calibration curve and is represented as the standard error (SE), derived from the division of the standard of deviation (SD) by the square-root of counts obtained in each gated bead population. The linear fit of the calibration curve was weighted with the SEM of each bead population. The slope and intercept of each calibration curve for the $500 \mathrm{~nm}$ Si FITC-MESF and $7 \mu \mathrm{m}$ PS FITC-MESF beads (Suppl. Fig. 2), was used to deduce the molecules of FITC equivalence for MLVsfGFP.

The virus population used for fluorescence quantification was identified based on its SSC and GFP fluorescence intensity and background fluorescence of the virus was subtracted using the fluorescence values of the gated MLVnoGFP. The mode of the sfGFP fluorescence intensities was used in determining the FITC-MESF value of MLVsfGFP. This statistic was chosen because it best represents the maximum of the unimodal distribution of our monodisperse virus population and is also the statistic most resistant to contributions from background noise events, which can be variable between day-to-day flow cytometer operations. The reported MFI and MESF values for MLVsfGFP was based on three separate experiments with a total of $\mathrm{n}=8$ and $\mathrm{n}=9$ samples. Calibration fits were produced using a $\mathrm{C}++$ macro compiled with ROOT under the general public license (https://root.cern.ch/downloading-root). The slope and intercepts from the calibration fits were inputted into FlowJo to display the data as a derived parameter in terms of MESF units.

Antibody labeling of MLV and MLV infected cells. For antibody labeling of MLV, the concentration of viral particles harvested from the supernatants of cells infected with MLVsfGFP and MLVnoGFP was adjusted to $10^{9}$ viral particles/ml for staining. Fluorophore-conjugated antibody aliquots were centrifuged at $17,000 \mathrm{xg}$ for $10 \mathrm{~min}$ prior to use to reduce the presence of aggregates. For each antibody labeling reaction, $50 \mu \mathrm{l}$ of virus supernatant was labeled with anti-GFP antibodies unconjugated or conjugated with PE, AF647 (clone FM264G, Bio Legend, San Diego, CA), or BV421 (clone 1A12-618), anti-mCD63-PE (clone NVG-2), anti-mCD81$\mathrm{PE}$ or BV421 (clone Eat2), or anti-mCD9 PE (clone $\mathrm{KMC}$, BD Biosciences, Mississauga, $\mathrm{ON}$ ) at the indicated concentrations for 1 hour at $37^{\circ} \mathrm{C}$ in a total volume of $100 \mu \mathrm{l}$. For titration of all anti-GFP antibodies, $5 \times 10^{7}$ MLVsfGFP viral particles were mixed with an equal number of MLVnoGFP particles, and a range of antibody staining concentrations from $0.0125 \mu \mathrm{g} / \mathrm{ml}$ to $1.6 \mu \mathrm{g} / \mathrm{ml}$ was tested for each anti-GFP conjugate. Unlabeled virus and antibody alone samples were run as controls for antibody labeling experiments. Labeled virus and controls were diluted 1:500 $\left(\sim 10^{6}\right.$ particles $\left./ \mathrm{ml}\right)$ for analysis in $0.1 \mu \mathrm{m}$-filtered PBS for analysis by FCM. For antibody labeling of MLV infected cells, $10^{6}$ cells were labeled with a concentration of $1 \mu \mathrm{g} / \mathrm{ml}$ of the same anti-tetraspanin antibodies used for MLV labeling in a $200 \mu \mathrm{l}$ staining volume of $0.2 \%$ BSA-PBS for $20 \mathrm{~min}$ at $4^{\circ} \mathrm{C}$. Excess antibody was removed by washing with $0.2 \%$ BSA-PBS. The SI was calculated for each anti-GFP conjugate at each concentration and the optimal staining concentrations associated with the highest SI value for anti-GFP PE, BV421, and AF647 were $0.2 \mu \mathrm{g} / \mathrm{ml}, 0.8 \mu \mathrm{g} / \mathrm{ml}$, and $0.4 \mu \mathrm{g} / \mathrm{ml}$ respectively. The SI is defined as the difference of the MFI of the stained MLVsfGFP and MLVnoGFP divided by the standard of deviation of MLVnoGFP.

To assess the expression of cell-derived tetraspanins on MLV, MLVsfGFP was labeled with anti-mouse CD9, CD63, or CD81 antibodies conjugated with PE because this fluorophore was found to produce the highest SI. Gating strategy used to identify tetraspanin stained vs. negative particles is shown in 
Tang et al., 2019

Supplementary Figure 3 (panels A \& B). Nonspecific labeling with rat IgG-PE on MLV occurs at antibody concentrations greater than $1.6 \mu \mathrm{g} / \mathrm{ml}$ (Suppl. Fig. 4A). Lower concentrations of each antibody were also tested and confirmed that the optimal staining concentration (highest SI) was indeed $1.6 \mu \mathrm{g} / \mathrm{ml}$ (Suppl. Fig. 3C). Virus was identified by SSC intensity and gated to remove antibody aggregates using the antibody-only control samples (Suppl. Fig. 5A: red gates, and 5B: red events). PE and GFP intensities of anti-tetraspanin PE labeled fluorescent virus was converted to MESF using QuantiBrite PE and 500nm Si FITC MESF beads. QuantiBrite PE beads were chosen in this case because there are no commercially available small particle PE MESF beads.

Nanoparticle Tracking Analysis. NTA was carried out as previously described (8). Briefly, samples were diluted with $0.1 \mu \mathrm{m}$-filtered PBS and analysed using the ZetaView PMX110 Multiple Parameter Particle Tracking Analyzer (Particle Metrix, Meerbusch, Germany) in diameter mode using ZetaView software version 8.02.28. Camera gain: 938, Shutter: 70, Frame Rate $30 \mathrm{fps}$, Temperature 24.5, Brightness: 30. Videos were taken from all 11 camera positions.

Light scatter modelling using Mie Scatter. Effective RI was approximated using the scatter-diameter curves based on the CytoFLEX S collection geometry as previously published (31). Briefly, instrument light scatter calibration was performed by fitting acquired $405 \mathrm{~nm}$ light scatter data to predicted values using the reported diameters and refractive indices of Apogee Mix beads (Apogee info) and NISTtraceable beads (Beckman Coulter, Brea, CA). These ranged in diameter from $80 \mathrm{~nm}$ polystyrene to 1300 nm silica (Fig. 1C).

Data Sharing. All List-mode data files have been made available on FlowRepository.org in compliance MIFlowCyt Checklist item 4.1. Repository ID: http://flowrepository.org/id/FR-FCM-Z24Y.

\section{RESULTS}

MLV virions are monodisperse. The ecotropic Moloney MLV used for this study was modified to prevent the expression of the membrane-associated accessory glycogag protein (27). This alteration ensured that the only viral protein expressed on its surface is Env. This virus is termed MLVnoGFP in our study. MLVsfGFP consists on this same virus but with the insertion of sfGFP in the Env protein sequence. MLV virions were detected as a highly monodisperse population that could be resolved by side-scatter (SSC) intensity alone and further identified by GFP expression (Fig. 1A, red gates). Next, the 405-SSC intensity of the virus was compared to two types of sizing beads: CytoFLEX Sizing Mix (prototype, Beckman Coulter) (Fig. 1B) and ApogeeMix (Fig. 1C). The virus gate (red) from Figure $1 \mathrm{~A}$ was superimposed to panels $1 \mathrm{~B}$ and $1 \mathrm{C}$ to delineate where the fluorescent MLV population would appear with reference to the bead populations on our instrument system. MLV has a similar SSC intensity to $80 \mathrm{~nm}$ polystyrene beads (Fig. 1D). A comparison of the standard deviation (SD) in diameter distribution of MLVsfGFP and $100 \mathrm{~nm}$ polystyrene bead by nanoparticle tracking analysis (NTA) show a greater variability in diameter sizes in the beads versus virus, 44.3 and 32.9 , respectively (Fig. 1E). This reflects the homogeneous and consistent stoichiometry of virus assembly, and suggests that formation of monodisperse MLVs is more consistent than the manufacturing methods currently used for production of NIST-traceable $100 \mathrm{~nm}$ polystyrene beads.

Fluorescence quantification and enumeration of GFP molecule expression on MLVsfGFP. The fluorescence signal was analyzed from MLVsfGFP viral particles, using MLVnoGFP as the autofluorescence control and MESF calibration beads for fluorescence quantification (Fig. 2A). $500 \mathrm{~nm}$ silica spheres containing known MESF values of fluorescein isothiocyanate (500 nm Si FITC-MESF) (30) were used in lieu of GFP given that GFP-MESF beads in the relevant diameter and fluorescenceintensity range are currently not commercially available. The GFP intensity expressed by MLVsfGFP, quantified using the 500nm Si FITCMESF beads, was found to be $637 \pm 3$ FITCequivalent molecules. Due to the mismatch of fluorophores between the FITC-MESF calibration beads and the MLVsfGFP, we could not report the 
Tang et al., 2019

fluorescence intensity of the virus in terms of GFPMESF. Env-sfGFP expression was therefore quantified on MLV by an alternate method using anti-GFP-PE antibody labeling and fluorophorematched QuantiBrite PE beads (Fig. 2B). A titration of the anti-GFP-PE antibody was performed to determine the concentration that would produce optimal labeling of Env-sfGFP using MLVnoGFP as an internal non-specific binding control (Fig. 4B and 4E). The brightest population of QuantiBrite PE beads were off-scale and only the first three populations were used. Anti-GFP-PE labeled MLVsfGFP had a PE-MESF value of $306 \pm 13$, corresponding to 102 Env spikes. However, this quantification method also has potential limitations because it could underestimate the expression level of Env-sfGFP for several reasons: 1) inaccuracies associated with the use of MESF beads that are not calibrated for use with small particles, 2) quenching of PE molecules due to the proximity of target epitopes, 3) steric hindrance could prevent binding of all available epitopes, and 4) the bivalent nature of the antibody.

Cross-institution and cross-platform assessment of fluorescence quantification. To compare the impact of 1) instrument variability, 2) user data acquisition variability, and 3) technological platform variations on the consistency of fluorescence quantification of the viruses, MLVsfGFP and MLVnoGFP viruses were sent to two different research institutions. The first institution operated a Beckman Coulter CytoFLEX S (Inst. 1) where virus fluorescence was quantified using $7 \mu \mathrm{m}$ PS FITCMESF beads (Fig. 3A). The second institution (Inst 2), operated a Luminex ImageStream X (ISX) where fluorescence quantifications were performed using $500 \mathrm{~nm}$ Si FITC-MESF beads (Fig. 3B). The values for FITC-MESF obtained on the sfGFP-expressing virus by Inst. 1 were very similar to our own, within a 0.25 -fold difference, while Inst. 2 (ISX) produced values that were 2.6-fold higher (Fig. 3C). This apparent disparity was most likely due to differences in spectral filters between the two platforms. The width of the 525/40 bandpass filter used in the CytoFLEX S for collection of signal from FITC and
GFP limits the collection of emitted photons to $62.7 \%$ and $59.2 \%$, respectively (Fig. 3D and 3E). The wider filter on the ISX $(520 / 80)$ is collecting $83.3 \%$ and $88.9 \%$ of photons emitted from FITC and GFP, respectively. This would suggest that the ISX was disproportionately collecting more signal from GFP than FITC (1.5-fold more compared to 1.3-fold), which could contribute to MLVsfGFP appearing brighter with respect to the FITC-MESF beads, highlighting a potential caveat of using mismatched fluorophores for fluorescence quantification.

Antibody labeling of MLV surface antigens. FCM is the preferred method for immunophenotyping of cells. However, immunophenotyping of small particle populations, such as EVs and viruses, is inherently challenging due to low surface antigen abundance as a result of their restricted surface area. For optimal resolution of these populations, fluorophore selections are therefore limited to the brightest options, with minimal spectral spillover, thereby reducing the number of antigens that can be targeted in one antibody panel. In cells, fewer than 1000 molecules/cell is considered low antigen abundance (32). According to our own measurements, Env-sfGFP expression on MLV is potentially in the order of $10^{2}$ molecules (Fig. 2). Compared to a cell, this may seem low in total abundance, yet when integrated over surface area, this amount of antigen on a nanoparticle of $\sim 100 \mathrm{~nm}$ in diameter actually translates to very high antigen density; the equivalent of several millions of molecules on a $10 \mu \mathrm{m}$ cell. As a result, labeling of small particles with high antigen density could potentially present the challenge of steric hindrance issues that may occur for antibodies conjugated to larger fluorophores.

Many factors contribute to the number of photons detected by a flow cytometer from a fluorescentlylabeled particle. These factors include: excitation wavelength, spectral filters, quantum efficiencies of detectors at increasing wavelengths, and the fluorophore to protein ratio (F:P ratio) of antibodies used to label the particles of interest. For the purposes of our study, instrument-specific 
Tang et al., 2019

considerations, such as excitation wavelength and spectral filters, are negated since analyses were performed on the same instrument. Avalanche photodiodes (APDs), the detectors used in the CytoFLEX S, also have a similar quantum efficiency over the range of visible light (400-800 nm) (33). The F:P ratio of conjugated antibodies, however, is a factor that should be considered, aside from the brightness, when choosing fluorophore conjugates since it is affected by the size of the fluorophore. Larger fluorophores such as PE typically have a 1:1 ratio due to steric hindrance, whereas smaller fluorophores could have a higher F:P ratio (34). Hence, a particle labeled with an antibody conjugated to the brightest fluorophore maybe not necessarily result in the greatest number of photons detected if the F:P ratio is low.

To assess the contributions of fluorophore size and brightness to resolving MLVsfGFP, an antibody against the high-density Env-sfGFP surface antigen was tested. Three different fluorophores that range in diameter and emission spectra, conjugated to an anti-GFP antibody were tested: PE, Brilliant Violet 421 (BV421), and Alexa Fluor 647 (AF647). The characteristics of each fluorophore, including brightness $(\varepsilon \phi)$ and size $(\mathrm{kDa})$, are summarized in Figure 4A. PE is the largest and brightest of the three fluorophores, followed by BV421, and AF647. A titration was performed for the three conjugates of anti-GFP antibodies and the stain index (SI) was calculated for each (Fig. 4B to 4E). At optimal staining concentrations (highest SI), both the PE and BV421 conjugates identified an equivalent frequency of $\mathrm{GFP}^{+}$viruses (52\%), while the AF647 conjugate labeled slightly fewer $\mathrm{GFP}^{+}$viruses than the other two fluorophore conjugates (46\%) (Fig. 4F). As with labeling of cells, increase of the cell or particle concentration will decrease the SI of optimized antibody concentrations. We confirmed that at the optimal staining concentration of $0.2 \mu \mathrm{g} / \mathrm{ml}$ for antiGFP PE, increasing the particle concentration of the sample does indeed decrease the SI, however this was only observed when particle concentrations increased by more than a factor of 2 (Suppl. Fig. 4B and $4 \mathrm{C}$ ). We also observed that staining saturation is reached for the MLVsfGFP virus at a concentration of $1.6 \mu \mathrm{g} / \mathrm{ml}$ of the anti-GFP antibody (Suppl. Fig. $4 \mathrm{~A})$.

Although PE is a very bright fluorophore, one major caveat in using PE-conjugated antibodies for small particle FCM is its potential to form aggregates (35). In fact, $\mathrm{PE}^{+}$particles were detected in samples containing only anti-GFP PE antibody which increased in number with rising concentrations of antibody used (Suppl. Fig. 5A). The majority of these $\mathrm{PE}^{+}$aggregates were located in two populations (coloured events); one lower and the other higher than the labeled virus in SSC intensity (gray events) (Suppl. Fig. 5B). At the optimal staining concentration of $0.2 \mu \mathrm{g} / \mathrm{ml}$ for anti-GFP PE, the number of aggregates was negligible in comparison to the number of stained particles (Suppl. Fig. 5A \& B). However, it is important to note that these samples were stained at $0.2 \mu \mathrm{g} / \mathrm{ml}$, but then further diluted 1:500 for analysis, resulting in an actual antibody concentration of $0.4 \mathrm{ng} / \mathrm{ml}$ when analysed on the flow cytometer. Aggregates can also be seen with the anti-GPF BV421 conjugate, but not with the AF647 conjugate (Suppl. Fig. 5C and D).

Antibody labeling of MLV modulates scatter intensity, hydrodynamic diameter, and the refractive index. During the analysis of our antibody-labeled MLVs in the previous section, we noted that the $\mathrm{GFP}^{+}$virus populations increased in SSC intensity with increasing amounts of anti-GFP $\mathrm{PE}$ antibody (Fig. 5A; red gates). This increase in SSC was also observed with BV421 and to a lesser extent the AF647 conjugate (Fig. 5B). Conceptually, it is feasible that labeling with antibodies could significantly increase the apparent diameter of a small particle such as MLV. The diameter of an IgG antibody has been reported to range from 14 to 40 $\mathrm{nm}$ in diameter by 2 to $4 \mathrm{~nm}$ in height depending on the measurement method used $(36,37)$. IgG conjugated with $\mathrm{PE}$, which is $250 \mathrm{kDa}$ and considered one of the larger fluorophores used in flow cytometry, has been reported to measure $60 \mathrm{~nm}$ in diameter by $5 \mathrm{~nm}$ in height by atomic force microscopy (35). To determine if the increase in SSC 
Tang et al., 2019

intensity is due to an increase in particle diameter, NTA was used to determine the hydrodynamic diameter of antibody-labeled MLVsfGFP at the oversaturating concentration of $1.6 \mu \mathrm{g} / \mathrm{ml}$. The median particle diameter and distribution were compared for unstrained MLVsfGFP and MLVsfGFP labeled with unconjugated anti-GFP, as well as PE, BV421, and AF647-conjugated antibodies (Fig. 5C). A scatter-modeling program based on Mie theory was used to calibrate the SSC intensity, to relate the SSC intensity to the measured hydrodynamic diameters of antibody-labeled and unlabeled MLVsfGFP determined with NTA (38), and to infer the RI. Data on the $405 \mathrm{~nm}$ scatter intensities acquired from polystyrene $(\mathrm{RI}=1.6333)$ and silica $(\mathrm{RI}=1.448)$ beads of known diameter (NIST-traceable) were used for calibration of our instrument (Fig. 1B). Our analyses showed high correlation $\left(\mathrm{R}^{2}=0.9999\right)$ of acquired values (geometric symbols) with theoretical values (solid lines) down to $80 \mathrm{~nm}$ for PS (Fig. 5D). Theoretical lines represent Mie-theory simulations for materials of specific RIs with increasing particle diameter, scatter intensity, and scattering crosssection. Measured values for the diameters and scatter intensities of particles with the same RI are predicted to fall on the same lines as seen with the PS, fluorescent PS (FL PS), and Si beads (Fig. 5D). Figure 5E, generated from the gray inset in Figure $5 \mathrm{D}$, depicts the collected data of antibody labeled MLV with respect to the RI values for PS (solid blue line) and Si (dashed red line).

Although individual MLV particles are not homogeneous in composition like a bead, their effective $R I$ was calculated with this assumption to simplify the modeling of particles with multiple refractive indices due to mixed compositions (Fig. $5 F)$. Here, the effective RI assumes the scattering intensity of each particle is related only to its refractive index and has no contributing extinction coefficient, as seen with fluorescent polystyrene beads (Fig. 5E). The dotted line, which passes through the unstained MLVsfGFP represents the effective RI of unstained virus (RI=1.519). The SSC intensity of antibody-labeled viruses falls below the iso-RI line of the unlabeled virus, indicating that labeled viruses have a lower effective RI than unlabeled virus. These results clearly show that antibody labeling can increase the diameter and, interestingly, reduces the effective RI, and therefore light scattering properties, of small particles. This may be related to the extinction coefficient of the fluorophore conjugated antibodies.

Quantification of host cell-derived tetraspanins on MLV. Host-derived antigen expression on the surface of the virus by antibody labeling was next assessed to determine whether the observations from anti-Env-sfGFP labeling held true for other antigens on the surface of the virus. We chose to target cellderived tetraspanins on the surface of MLV because these transmembrane glycoproteins are ubiquitously expressed as they contribute to fundamental processes of cellular trafficking (39). Tetraspanins CD9, CD63, and CD81 have been used as markers to identify subtypes of EVs due to their association with mechanisms of EV egress, such as the endosomal sorting complexes required for the transport (ESCRT) pathway (39). More specifically, these pathways have also been implicated in both cellular entry and egress of retroviruses (40-44).

The PE MESF of anti-tetraspanin-labeled viral particles were compared to show the relative expression levels of CD9, CD63, and CD81 on MLV (Fig. 6A-E). This comparison is possible because, in contrast to other fluorophores, only one PE molecule is likely to be conjugated per IgG due to its large diameter (34). CD81 was most abundantly expressed on MLVsfGFP with a median PE MESF of $18.7 \pm 0.2$, followed by CD63 with 13.1 \pm 0.3 PE MESF, and CD9 with 6.4 $\pm 0.02 \mathrm{PE} \mathrm{MESF}$. It is important to note that these values should be taken as a measure of relative tetraspanin abundance between the three types, and not as actual molecules of tetraspanins expressed per virus, since QuantiBrite PE beads were not intended for use with such dimly expressed antigens and so are not accurately calibrated for this purpose (45). It is unclear whether CD9 expression was actually present on MLVsfGFP since the signal was similar to unstained virus (3.6 $\pm 0.02 \mathrm{PE}$ MESF) and could potentially be the result of non-specific labeling. 
Tang et al., 2019

However, CD9 was confirmed to be expressed on the cells producing MLVsfGFP, therefore it is possible that MLVsfGFP indeed express CD9 at very low levels, below the detection limit of our flow cytometer (Suppl. Fig. 3D).

The level of staining produced by the same antiCD81 antibody conjugated to PE vs. BV421 was compared (Fig. 6F). Labeling of MLVsfGFP with anti-GFP-PE resulted in a higher SI than with the BV421 conjugate, although both equally resolved the MLVsfGFP population at optimal staining concentrations (Fig. 4B, 4C, and 4F). At optimal staining concentrations, labeling MLVsfGFP with anti-CD81-PE resulted in approximately $20 \%$ higher frequency of $\mathrm{CD} 81^{+} \mathrm{GFP}^{+}$viruses than anti-CD81BV421 (Fig. 6G). The resolution of CD81 expression, an antigen expressed at lower levels than Env-sfGFP, benefited significantly from the use of a brighter fluorophore.

To assess whether double-labeling, the targeting of two different antigens with two different antibodies, would result in reduced staining for each individual antigen due to possible steric hindrance between the fluorophore-conjugated antibodies. A comparison was made between the percent of resulting $\mathrm{CD} 1^{+} \mathrm{GFP}^{+}$and $\mathrm{CD}^{+}{ }^{+} \mathrm{GFP}^{+}$viruses (based on the gating strategy used in Fig. 6F; dashed gates) when the virus was labeled with anti-CD63 PE and antiCD81 BV421 individually or with both antibodies together. There was no significant difference between the numbers of CD $81^{+}$MLVsfGFP or CD63 ${ }^{+}$MLVsfGFP events, or in the percentage of $\mathrm{CD} 1^{+}$or $\mathrm{CD}^{+} 3^{+} \mathrm{GFP}^{+}$events, obtained using single versus double-labeling (Fig. $6 \mathrm{H}$ and 6I). This suggests that steric hindrance did not affect in this case the individual binding of two antibodies targeting distinct antigens.

SSC intensities for single and double-labeled virus populations (Fig. 6J) were compared to determine if fluorescence labeling of lower-density antigens would similarly impact scatter intensity and, thus, the apparent diameter and RI. Although there was an appearance of a correlation between the highest SSC intensities and the highest degree of labeling $(\mathrm{CD} 81+\mathrm{CD} 63>\mathrm{CD} 81>\mathrm{CD} 63>\mathrm{CD} 9)$, these values were not statistically different from those of the unlabeled virus. Therefore, these observations suggest that the labeling of low-density antigens with fluorophore-conjugated antibodies does not significantly alter the scatter intensities of small particles.

\section{DISCUSSION}

Current challenges for the analysis of small biological particles by flow cytometry are multi-faceted. To reliably achieve single-molecule resolution, technological advancements are needed to further improve instrument sensitivity. Development of brighter and smaller fluorophores is required for multi-parameter analyses of small particles where surface area is highly restricted. But more urgently, different types of reference particles with low fluorescence levels relevant to small particles are also needed for: 1) positive controls for stainings (antibodies and dyes), and 2) instrument calibration to allow standardized data reporting across instruments and technological platforms.

At present, few references particles are available for small particle FCM. Many FCM reference beads, such as compensation beads, are made mostly of polystyrene and exhibit fluorescence and autofluorescence intensity levels that are much higher than those attainable with small biological particles. Chemical conjugation of biological molecules to synthetic beads for use as positive controls can be technically challenging and often result in very high levels of expression that are biologically irrelevant. Currently available calibration beads are also too large and too bright for accurate small particle fluorescence standardization using MESF axes calibration. Biological particles, such as retroviruses, on the other hand, have long been adapted for use as vectors to safely express proteins of interest in cells. MLV particles are small, monodisperse, and have minimal autofluorescence. The potential of MLVs for use as fluorescence calibration particles in FCM is obvious because they 
Tang et al., 2019

can be engineered or labeled to have multiple levels of fluorescence expression. We showed here, with titrating levels of anti-GFP antibody, that MLVsfGFP can be easily labeled with different intensities of fluorescence.

In this study, MLV was engineered to express sfGFP in fusion with the viral surface glycoprotein, Env. These virus particles were used to showcase the importance of FCM best practices, such as antibody titration and fluorophore selection, when conducting immunophenotyping assays on small biological particles. Additionally, we demonstrated that when a highly-expressed surface antigen on a small particle is labeled with an excess of fluorophore-conjugated antibodies, these can change the physical properties, including the diameter and effective RI of that small particle. Additionally, this study emphasized the importance of fluorescence standardization with matching fluorophores to compare data between different flow cytometry platforms. Taken together, our observations on antibody labeling using MLV as a prototypical small particle, enabled us to identify and address specific challenges relevant to the antibodylabeling of small biological particles. These observations were only made possible due to the stringent uniformity in diameter and fluorescence, and high viral surface antigen expression on MLV particles. These critical features decisively qualify MLV as a candidate biological reference particle for the FCM analysis of other enveloped viruses and small biological particles such as EVs.

\section{ACKNOWLEDGEMENTS}

The authors would especially like to thank members of the Beckman Coulter technical support team, Dominic Therrien and Marc Simard, for valuable assistance throughout this study. We would like to acknowledge Sergei Gulnik and Maria Gentile of the Beckman Coulter Research and Marketing Teams for their helpful discussions. We would like to thank Christian Ouellet for providing the $\mathrm{C}++$ scripts for MESF calibration. V.A.T. is an International Society for Advancement of Cytometry (ISAC) Shared Resource Lab Emerging Leader. T.M.R. holds a Queen Elizabeth II Graduate Scholarship in Science and Technology (QEII- GSST). E.v.d.P. was supported by the Netherlands Organization for Scientific Research-Domain Applied and Engineering Sciences (NWO-TTW), research programs VENI 15924. M.-A.L. holds a Canada Research Chair in Molecular Virology and Intrinsic Immunity. This work was supported by a research and development grant from the University of Ottawa Faculty of Medicine to the FCV Core Facility, and by a Discovery Grant and an Idea to Innovation (I2I) Grant by the Natural Sciences and Engineering Research Council of Canada (NSERC) to M.-A.L.

\section{CONFLICTS OF INTERESTS}

M.-A.L. is the CEO, and V.A.T. is the CSO of ViroFlow Technologies. G.C.B is a Beckman Coulter research scientist. E.v.d.P. is CSO of Exometry.

\section{REFERENCES}

1. Hercher M, Mueller W, Shapiro HM. Detection and discrimination of individual viruses by flow cytometry. J Histochem Cytochem 1979;27:350-2.

2. Sanders CA, Yajko DM, Nassos PS, Hyun WC, Fulwyler MJ, Hadley WK. Detection and analysis by dual-laser flow cytometry of bacteriophage T4 DNA inside Escherichia coli. Cytometry 1991;12:167-71.

3. Steen HB. Flow cytometer for measurement of the light scattering of viral and other submicroscopic particles. Cytometry A 2004;57:94-9.

4. Lian H, He S, Chen C, Yan X. Flow Cytometric Analysis of Nanoscale Biological Particles and Organelles. Annu Rev Anal Chem (Palo Alto Calif) 2019.

5. Marcoux G, Duchez AC, Cloutier N, Provost P, Nigrovic PA, Boilard E. Revealing the diversity of extracellular vesicles using high-dimensional flow cytometry analyses. Sci Rep 2016;6:35928.

6. Lippe R. Flow Virometry: a Powerful Tool To Functionally Characterize Viruses. J Virol 2018;92.

7. Nolan JP, Duggan E. Analysis of Individual Extracellular Vesicles by Flow Cytometry. 
Tang et al., 2019

Methods Mol Biol 2018;1678:79-92.

8. Tang VA, Renner TM, Fritzsche AK, Burger D, Langlois MA. Single-Particle Discrimination of Retroviruses from Extracellular Vesicles by Nanoscale Flow Cytometry. Sci Rep 2017;7:17769.

9. Bonar MM, Tilton JC. High sensitivity detection and sorting of infectious human immunodeficiency virus (HIV-1) particles by flow virometry. Virology 2017;505:8090.

10. Musich T, Jones JC, Keele BF, Jenkins LM, Demberg T, Uldrick TS, Yarchoan R, Robert-Guroff M. Flow virometric sorting and analysis of HIV quasispecies from plasma. JCI Insight 2017;2:e90626.

11. Cloutier N, Tan S, Boudreau LH, Cramb C, Subbaiah R, Lahey L, Albert A, Shnayder R, Gobezie R, Nigrovic PA and others. The exposure of autoantigens by microparticles underlies the formation of potent inflammatory components: the microparticle-associated immune complexes. EMBO Mol Med 2013;5:235-49.

12. Lannigan J, Erdbruegger U. Imaging flow cytometry for the characterization of extracellular vesicles. Methods 2017;112:5567.

13. van der Pol E, de Rond L, Coumans FAW, Gool EL, Boing AN, Sturk A, Nieuwland R, van Leeuwen TG. Absolute sizing and labelfree identification of extracellular vesicles by flow cytometry. Nanomedicine 2018;14:801-810.

14. Welsh JA, Holloway JA, Wilkinson JS, Englyst NA. Extracellular Vesicle Flow Cytometry Analysis and Standardization. Front Cell Dev Biol 2017;5:78.

15. Parks DR, Moore WA, Brinkman RR, Chen Y, Condello D, El Khettabi F, Nolan JP, Perfetto SP, Redelman D, Spidlen J and others. Methodology for evaluating and comparing flow cytometers: A multisite study of 23 instruments. Cytometry A 2018;93:1087-1091.

16. Nolan JP. Flow Cytometry of Extracellular Vesicles: Potential, Pitfalls, and Prospects. Curr Protoc Cytom 2015;73:13 14 1-16.

17. Yeager M, Wilson-Kubalek EM, Weiner SG,
Brown PO, Rein A. Supramolecular organization of immature and mature murine leukemia virus revealed by electron cryo-microscopy: implications for retroviral assembly mechanisms. Proc Natl Acad Sci U S A 1998;95:7299-304.

18. Kozak CA. Origins of the endogenous and infectious laboratory mouse gammaretroviruses. Viruses 2014;7:1-26.

19. Wurdinger T, Gatson NN, Balaj L, Kaur B, Breakefield XO, Pegtel DM. Extracellular vesicles and their convergence with viral pathways. Adv Virol 2012;2012:767694.

20. Nolte-'t Hoen E, Cremer T, Gallo RC, Margolis LB. Extracellular vesicles and viruses: Are they close relatives? Proc Natl Acad Sci U S A 2016;113:9155-61.

21. Liu J, Bartesaghi A, Borgnia MJ, Sapiro G, Subramaniam S. Molecular architecture of native HIV-1 gp120 trimers. Nature 2008;455:109-13.

22. Kwong PD, Wyatt R, Robinson J, Sweet RW, Sodroski J, Hendrickson WA. Structure of an HIV gp120 envelope glycoprotein in complex with the CD4 receptor and a neutralizing human antibody. Nature 1998;393:648-59.

23. Stano A, Leaman DP, Kim AS, Zhang L, Autin L, Ingale J, Gift SK, Truong J, Wyatt RT, Olson AJ and others. Dense Array of Spikes on HIV-1 Virion Particles. J Virol 2017;91.

24. Zhu P, Chertova E, Bess J, Jr., Lifson JD, Arthur LO, Liu J, Taylor KA, Roux KH. Electron tomography analysis of envelope glycoprotein trimers on HIV and simian immunodeficiency virus virions. Proc Natl Acad Sci U S A 2003;100:15812-7.

25. Zhu P, Liu J, Bess J, Jr., Chertova E, Lifson JD, Grise H, Ofek GA, Taylor KA, Roux KH. Distribution and three-dimensional structure of AIDS virus envelope spikes. Nature 2006;441:847-52.

26. Butan C, Winkler DC, Heymann JB, Craven RC, Steven AC. RSV capsid polymorphism correlates with polymerization efficiency and envelope glycoprotein content: implications that nucleation controls morphogenesis. J Mol Biol 2008;376:1168- 
Tang et al., 2019

81.

27. Renner TM, Belanger K, Lam C, Gerpe MCR, McBane JE, Langlois MA. FullLength Glycosylated Gag of Murine Leukemia Virus Can Associate with the Viral Envelope as a Type I Integral Membrane Protein. J Virol 2018;92.

28. Schwartz A, Wang L, Early E, Gaigalas A, Zhang YZ, Marti GE, Vogt RF. Quantitating Fluorescence Intensity from Fluorophore: The Definition of MESF Assignment. J Res Natl Inst Stand Technol 2002;107:83-91.

29. Wood JC, Hoffman RA. Evaluating fluorescence sensitivity on flow cytometers: an overview. Cytometry 1998;33:256-9.

30. Zhang S, Zhu S, Yang L, Zheng Y, Gao M, Wang S, Zeng JZ, Yan X. High-throughput multiparameter analysis of individual mitochondria. Anal Chem 2012;84:6421-8.

31. van der Pol E, Sturk A, van Leeuwen T, Nieuwland R, Coumans F, group I-S-VW. Standardization of extracellular vesicle measurements by flow cytometry through vesicle diameter approximation. J Thromb Haemost 2018;16:1236-1245.

32. Balderas B. Optimization of Multicolor Panel Design Incorporating Low Receptor Density Antigens. BD Biosciences Webinar; 2013.

33. Hergert E. Detectors: Guideposts on the Road to Selection. 2019.

34. Davis KA, Abrams B, Iyer SB, Hoffman RA, Bishop JE. Determination of CD4 antigen density on cells: role of antibody valency, avidity, clones, and conjugation. Cytometry 1998;33:197-205.

35. Chen Y, Cai J, Xu Q, Chen ZW. Atomic force bio-analytics of polymerization and aggregation of phycoerythrin-conjugated immunoglobulin $\mathrm{G}$ molecules. Mol Immunol 2004;41:1247-52.

36. Hansma HG. Varieties of imaging with scanning probe microscopes. Proc Natl Acad Sci U S A 1999;96:14678-80.

37. Leatherbarrow RJ, Stedman M, Wells TN.
Structure of immunoglobulin $G$ by scanning tunnelling microscopy. J Mol Biol 1991;221:361-5.

38. van der Pol E, Coumans FA, Sturk A, Nieuwland R, van Leeuwen TG. Refractive index determination of nanoparticles in suspension using nanoparticle tracking analysis. Nano Lett 2014;14:6195-201.

39. Martin-Serrano J, Neil SJ. Host factors involved in retroviral budding and release. Nat Rev Microbiol 2011;9:519-31.

40. Nydegger S, Foti M, Derdowski A, Spearman P, Thali M. HIV-1 egress is gated through late endosomal membranes. Traffic 2003;4:902-10.

41. Molle D, Segura-Morales C, Camus G, Berlioz-Torrent C, Kjems J, Basyuk E, Bertrand E. Endosomal trafficking of HIV1 gag and genomic RNAs regulates viral egress. J Biol Chem 2009;284:19727-43.

42. Deneka M, Pelchen-Matthews A, Byland R, Ruiz-Mateos E, Marsh M. In macrophages, HIV-1 assembles into an intracellular plasma membrane domain containing the tetraspanins CD81, CD9, and CD53. J Cell Biol 2007;177:329-41.

43. Grigorov B, Attuil-Audenis V, Perugi F, Nedelec M, Watson S, Pique C, Darlix JL, Conjeaud H, Muriaux D. A role for CD81 on the late steps of HIV-1 replication in a chronically infected $\mathrm{T}$ cell line. Retrovirology 2009;6:28.

44. Martin-Serrano J, Yarovoy A, PerezCaballero D, Bieniasz PD. Divergent retroviral late-budding domains recruit vacuolar protein sorting factors by using alternative adaptor proteins. Proc Natl Acad Sci U S A 2003;100:12414-9.

45. Pannu KK, Joe ET, Iyer SB. Performance evaluation of QuantiBRITE phycoerythrin beads. Cytometry 2001;45:250-8. 

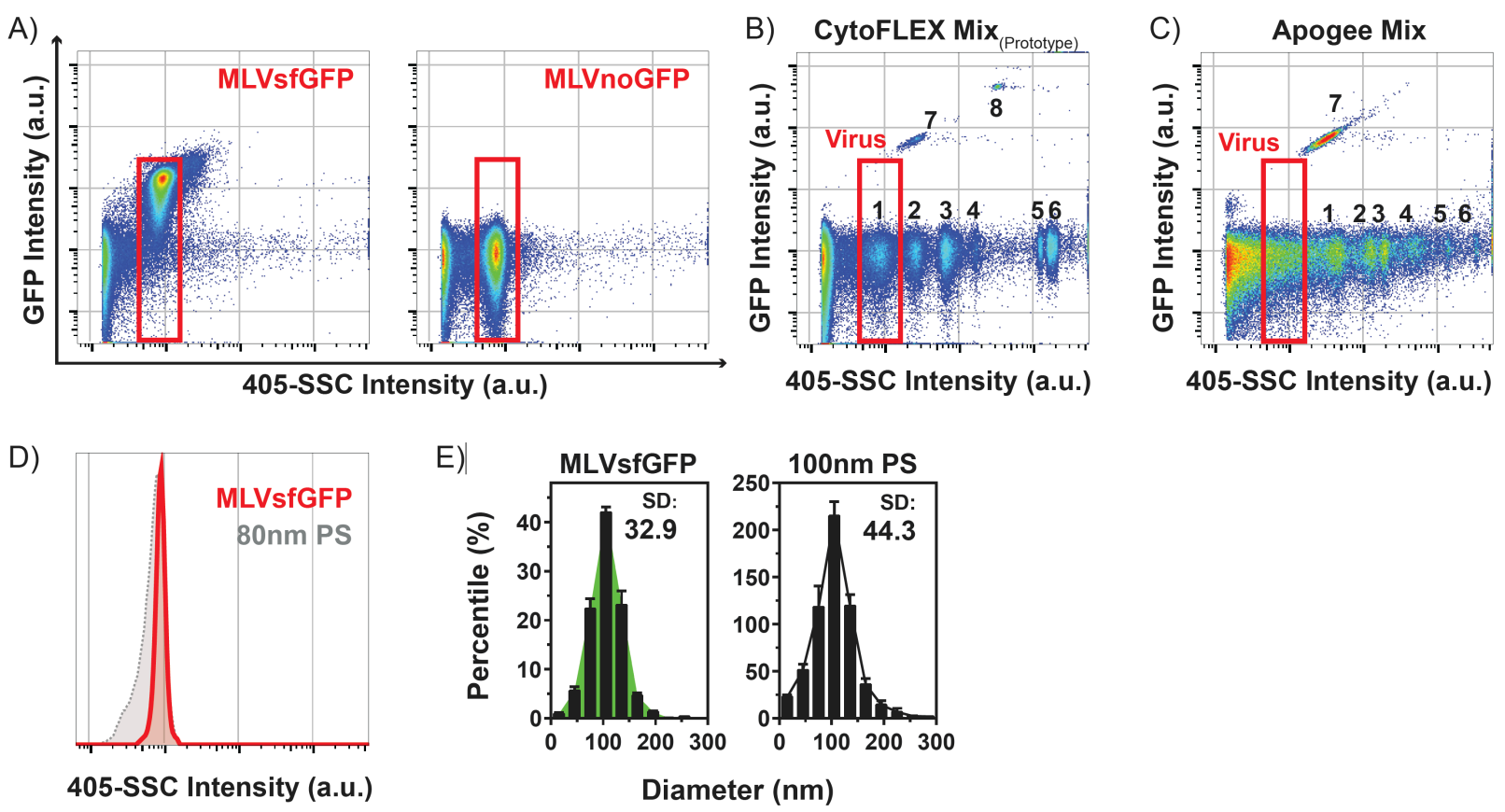

FIGURE 1. MLV virions are highly monodisperse. (A) MLVsfGFP and MLVnoGFP virions are a discrete population that can be resolved by 405-SSC intensity (a.u.) and green fluorescence intensity (a.u.). (B) Prototype CytoFLEX Sizing Mix (1: $80 \mathrm{~nm}$ PS, 2: $100 \mathrm{~nm}$ PS, 3: $214 \mathrm{~nm} \mathrm{Si}$, 4: 152nm PS, 5: $296 \mathrm{~nm}$ PS, 6: $1020 \mathrm{~nm} \mathrm{Si}, 7: 100 \mathrm{~nm}$ PS fluorescent, 8: $196 \mathrm{~nm}$ PS fluorescent) and (C) ApogeeMix (1: $180 \mathrm{~nm} \mathrm{Si}, 2: 240$ $\mathrm{nm} \mathrm{Si}, 3: 300 \mathrm{~nm} \mathrm{Si}, 4: 590 \mathrm{~nm} \mathrm{Si}, 5: 880 \mathrm{~nm} \mathrm{Si}, 6: 1300 \mathrm{~nm} \mathrm{Si}, 7: 110 \mathrm{~nm}$ PS fluorescent) were analysed using the same settings as those for the MLV viruses. The "Virus" gate in red is the same from panel A. (D) Comparison of the 405-SSC intensity (a.u.) of MLVsfGFP and $80 \mathrm{~nm}$ polystyrene (PS) beads. (E) NTA on the diameter distribution of $100 \mathrm{~nm}$ PS beads and MLVsfGFP; SD: standard deviation. 
Tang et al., 2019
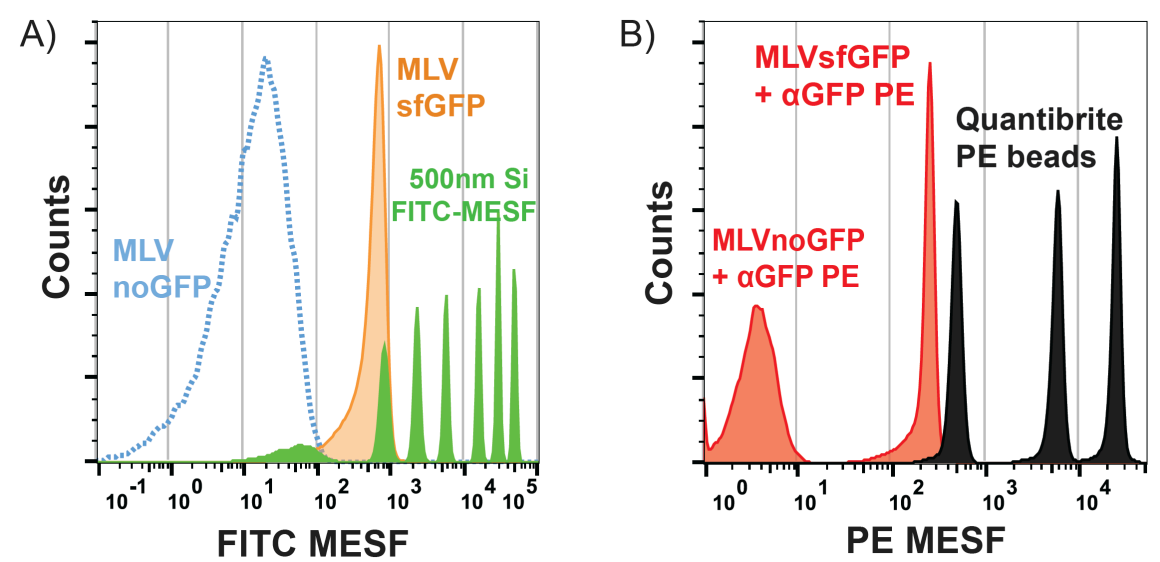

FIGURE 2. Fluorescence quantification of GFP expression on MLVsfGFP. (A) Green fluorescence from MLVsfGFP was quantified with 500nm Si FITC-MESF beads with MLVnoGFP as an autofluorescence control. Representative histogram overlay of MLVsfGFP and MLVnoGFP in FITC-MESF units with FITCMESF beads ( $n=5)$. (B) Fluorescence quantification of anti-GFP PE labeled MLVsfGFP with QuantiBrite PE beads using MLVnoGFP as an internal control for non-specific labeling $(n=3)$. 
Tang et al., 2019

A)

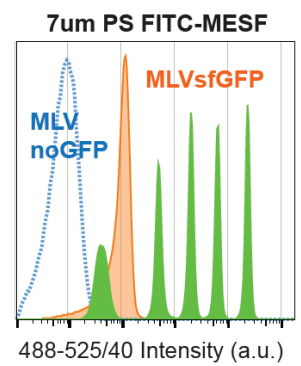

CytoFLEX (Inst 1)

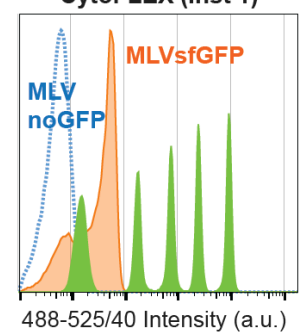

B)

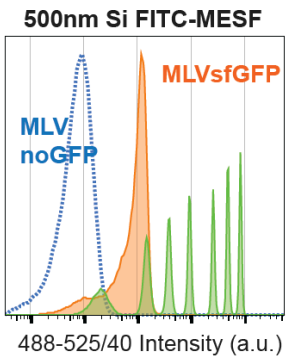

ISX (Inst 2)

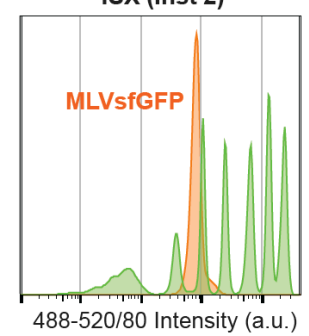

C)

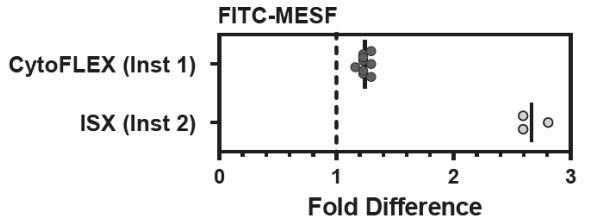

D)

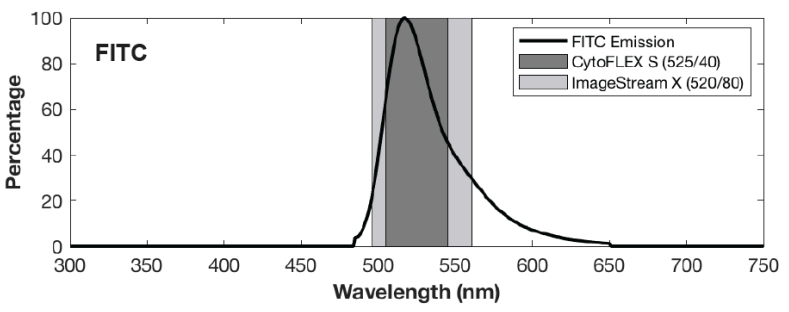

E)

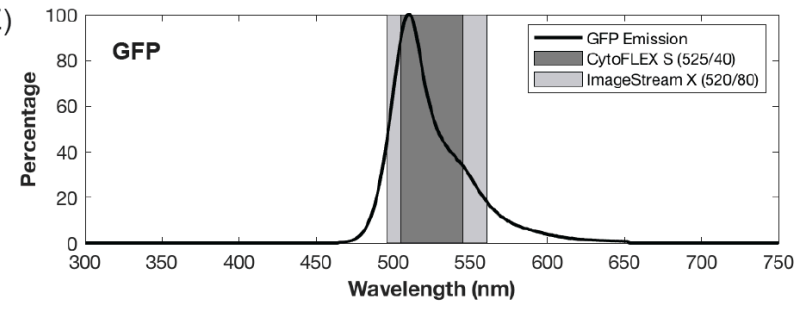

FIGURE 3. Cross-institution and cross-platform comparison of fluorescence intensity quantification of MLVsfGFP. (A) Comparative analysis of MLVsfGFP and MLVnoGFP viruses on two CytoFLEX S flow cytometers from two different institutions using $7 \mu \mathrm{m}$ PS FITC-MESF beads (top panel: uOttawa, bottom panel: Beckman Coulter (Inst1)). (B) Comparative analysis of MLVsfGFP and MLVnoGFP viruses on a Luminex ImageStream X (ISX) and a CytoFLEX S using 500nm Si FITC-MESF beads (top panel uOttawa CytoFLEX S, bottom panel uVirginia (Inst2) ISX). MLVnoGFP was not detected on the ISX. Data is displayed as fluorescence intensity. C) FITC-MESF values were calibrated for MLVsfGFP on both platforms and compared to values obtained with uOttawa CytoFLEX S, with uOttawa values set to "1" (dashed line). (D and E) Filter sets for the ISX and CytoFLEX $S$ that were used for detection overlaid with the emission spectrums of FITC (D) and GFP (E); CytoFLEX S: $n=9$; ISX; $n=3$. 
Tang et al., 2019

A)

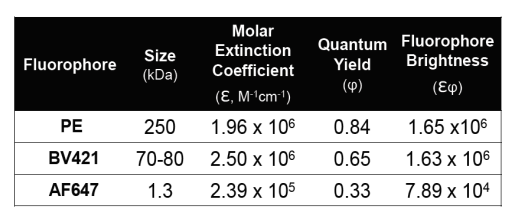

E)

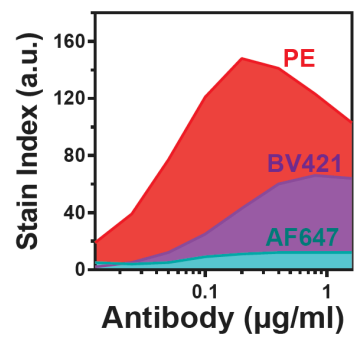

F)
B)

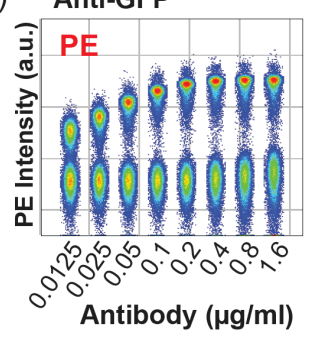

C)

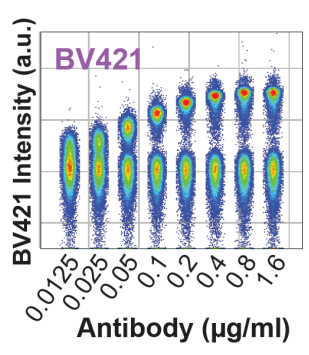

D)

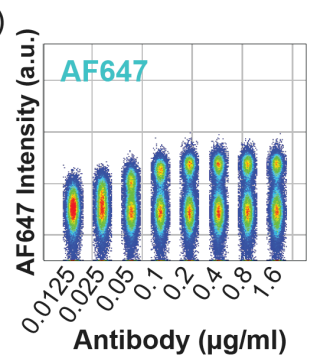

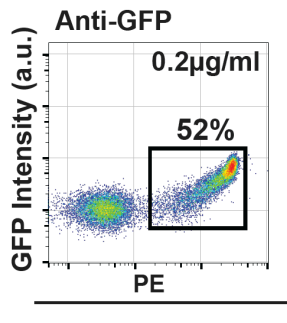

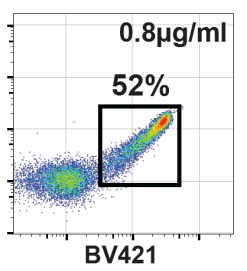

Intensity (a.u.)

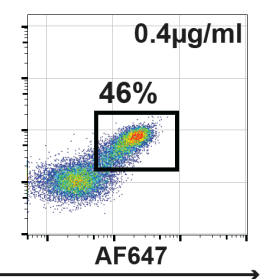

AF647

FIGURE 4. Evaluation of antibody-fluorophore conjugates for the detection of MLV. (A) Diameter and brightness information for PE, BV421, and AF647. Titration of anti-GFP (B) PE, (C) BV421, and (D) AF647 antibodies from $0.0125 \mu \mathrm{g} / \mathrm{ml}$ to $1.6 \mu \mathrm{g} / \mathrm{ml}$, performed on a mixture of equal proportions of MLVnoGFP and MLVsfGFP virus particles. (E) The SI, displayed is a representative graph of $n=6$, was calculated for each antibody at each concentration to determine the optimal staining concentration. (F) Representative dot-plots showing the frequency of anti-GFP ${ }^{+}$events labeled at optimal staining concentrations for each fluorophore conjugate. 
bioRxiv preprint doi: https://doi.org/10.1101/614461; this version posted June 7,2019 . The copyright holder for this preprint (which was not certified by peer review) is the author/funder, who has granted bioRxiv a license to display the preprint in perpetuity. It is made available under aCC-BY-ND 4.0 International license.

Tang et al., 2019

A)

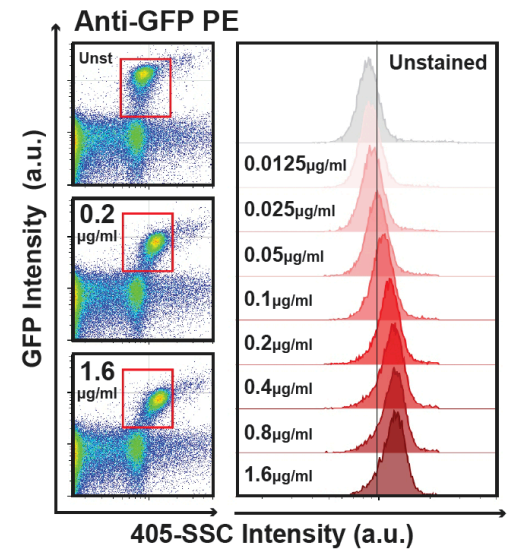

C)

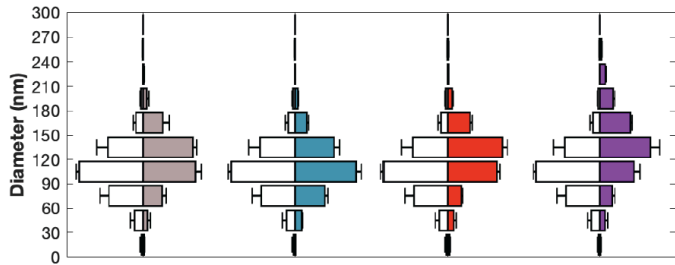

B)

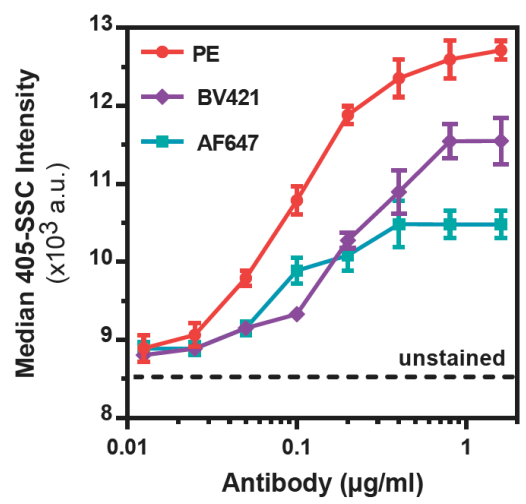

F)

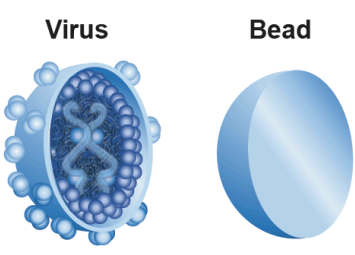

D)

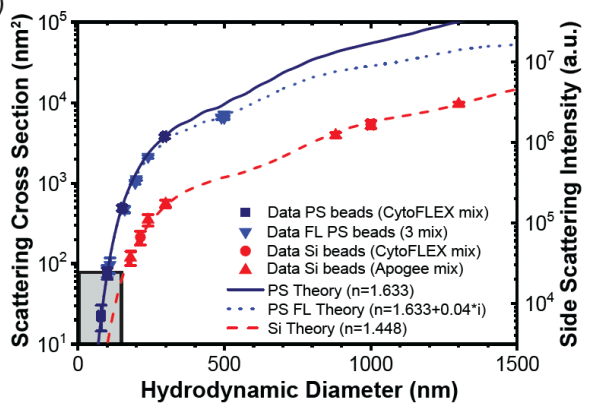

E)

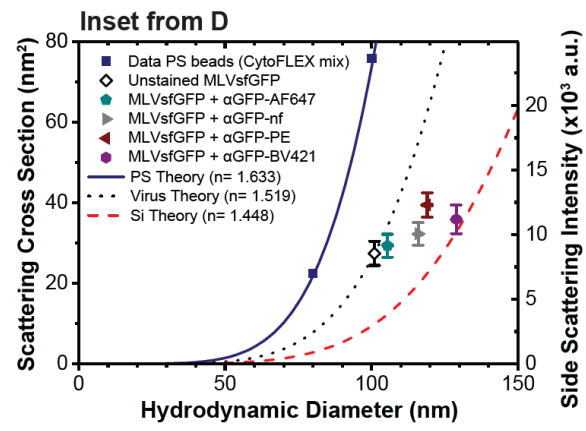

FIGURE 5. Antibody-fluorophore conjugates impact SSC intensity, hydrodynamic diameter, and effective refractive index of labeled viruses. (A) Antibody labeling of MLVsfGFP increases the SSC intensity of virus particles in a concentration-dependent manner. Representative scatter plots of MLVsfGFP (gated) mixed with MLVnoGFP that were unstained (Unst) or labeled with anti-GFP PE antibody at $0.2 \mathrm{ug} / \mathrm{ml}$ and $1.6 \mathrm{ug} / \mathrm{ml}$ (left panels). Histogram overlay of the gated $\mathrm{GFP}^{+}$populations that were labeled with anti-GFP PE, for all antibody concentrations from $0.0125 \mathrm{ug} / \mathrm{ml}$ to $1.6 \mathrm{ug} / \mathrm{ml}$ (right panel). (B) SSC intensity of $\mathrm{GFP}^{+}$viruses labeled with anti-GFP conjugated with PE, BV421, and AF647 at increasing antibody concentrations. Dashed line denotes SSC intensity of unstained MLVsfGFP. (C) NTA-measured diameter distribution of unstained MLVsfGFP separately compared to MLVsfGFP labeled with unconjugated anti-GPF, anti-GFP-PE, BV421, or AF647 at a concentration of $1.6 \mu \mathrm{g} / \mathrm{ml}$; $\mathrm{n}=3$. (D) Mietheory analysis for the calculation of the RIs for data points acquired using different silica (Si; red) and polystyrene (PS; blue) beads. The plot represents a correlation of the scattering cross section, hydrodynamic diameter, and SSC intensity of the virus particles. The gray-shaded box indicates the range where the MLV data points were acquired. (E) Gray-shaded box inset from (D), Mie-theory analysis of unstained MLVsfGFP and viruses labeled with various antiGFP conjugated antibodies. The estimated effective RI of unlabeled virus is demonstrated as a dotted black line; $\mathrm{n}=3$. (F) Cartoon to illustrate the complex and heterogeneous composition of a virus compared to the homogenous composition of a bead. 
bioRxiv preprint doi: https://doi.org/10.1101/614461; this version posted June 7, 2019. The copyright holder for this preprint (which was not certified by peer review) is the author/funder, who has granted bioRxiv a license to display the preprint in perpetuity. It is made available under aCC-BY-ND 4.0 International license.

Tang et al., 2019

A)

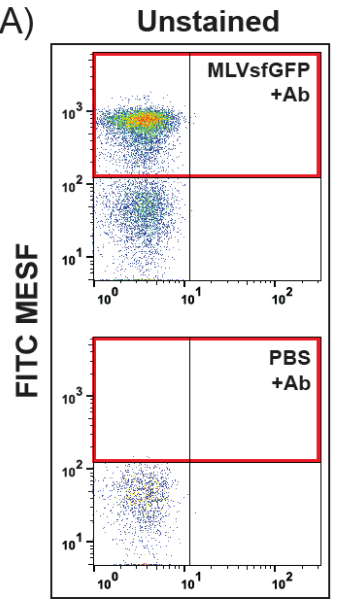

B)

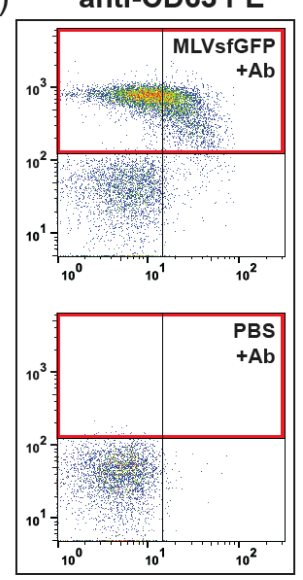

C)

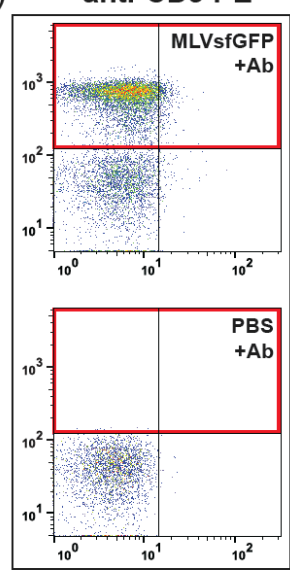

D)

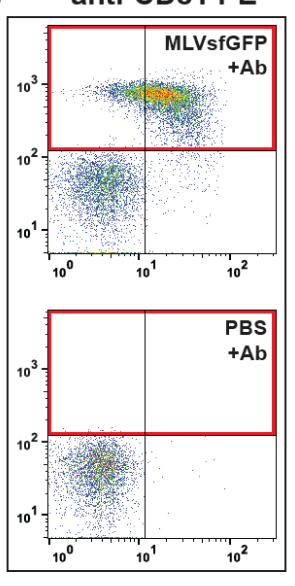

PE MESF

E)

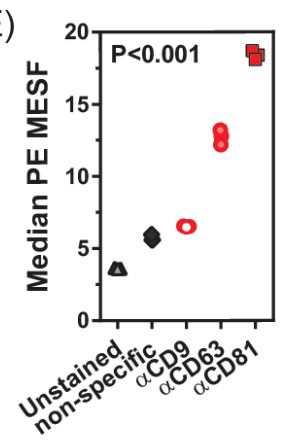

F)

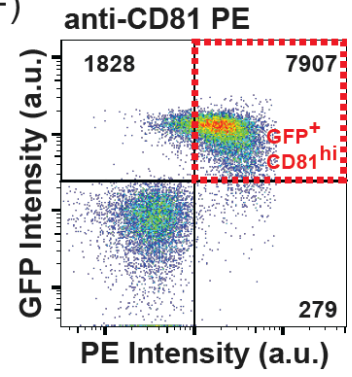

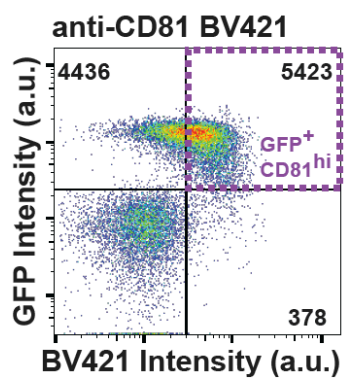

G)

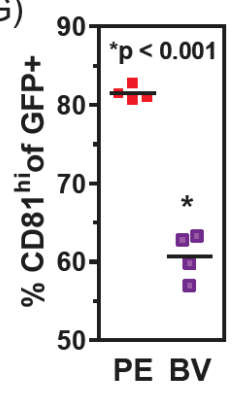

H)

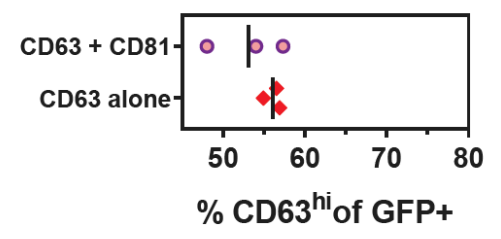

I)

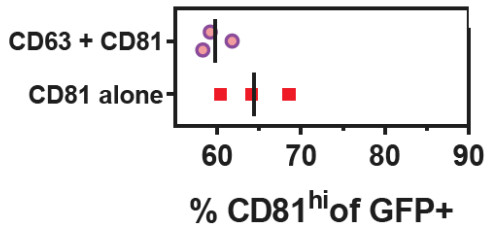

J)

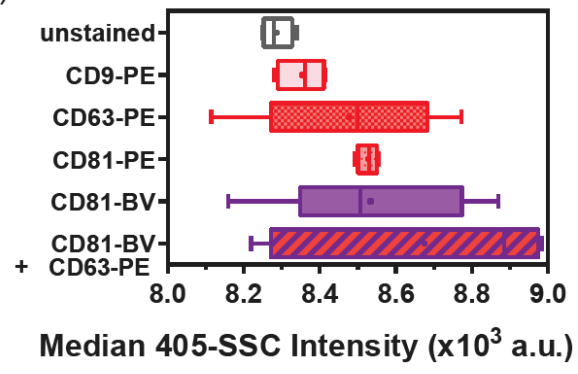

FIGURE 6. Phenotypic analysis of cell-derived tetraspanins expressed on the surface of MLV virions.

(A) Unstained MLVsfGFP was compared to virus labeled with (B) anti-CD63-PE, (C) anti-CD9-PE, and (D) antiCD81-PE at a concentration of $1.6 \mu \mathrm{g} / \mathrm{ml}$ of antibody per sample. QuantiBrite PE beads and 500nm Si FITC MESF were used to convert fluorescence intensity to PE and FITC MESF. (E) Median PE-MESF values of anti-tetraspanin labeled MLVsfGFP ( $\mathrm{n}=3$ ), 1-way ANOVA, $\mathrm{P}<0.001$. (F) Gating strategy for identification of $\mathrm{GFP}^{+} \mathrm{CD} 81^{\text {hi }}$ population from MLVsfGFP labeled with anti-CD81 BV421 and anti-CD81 BV421. (G) Comparison of GFP ${ }^{+} C D 811^{\text {hi }}$ events from anti-CD81 PE and anti-CD81 BV421 labeling, $n=3$, Unpaired t-Test, $\mathrm{p}<0.001$. (H) Comparison of the frequency of $\mathrm{CD}{ }^{\text {hi }}{ }^{\mathrm{GFP}^{+}}$virus in single- (anti-CD63 alone) vs. double-labeled (anti-CD63 + anti-CD81) viruses, Unpaired t-Test, non-significant, $\mathrm{p}=0.34$. (I) Frequency of CD81 ${ }^{\text {hi }} \mathrm{GFP}^{+}$virus in single- (anti-CD81 alone) vs. double-labeled (antiCD63 + anti-CD81) viruses, Unpaired t-Test, non-significant, $p=0.15$. (J) SSC intensities (405-SSC) for antitetraspanin labeled viruses, 1-way ANOVA, non-significant, $\mathrm{P}=0.16$. 


\section{SUPPLEMENTARY MATERIAL}

\section{Engineered Retroviruses as Fluorescent Biological Reference Particles for Small Particle Flow Cytometry}

Vera A. Tang ${ }^{1,2, *}$, Anna K. Fritzsche ${ }^{1,2}$, Tyler M. Renner ${ }^{2}$, Dylan Burger ${ }^{3}$, Edwin van der Pol ${ }^{4,5,6}$, Joanne A. Lannigan ${ }^{7}$, George C. Brittain', Joshua A. Welsh', Jennifer C. Jones', and Marc-André Langlois ${ }^{1,2,10, *}$ 
Tang et al., 2019

A)

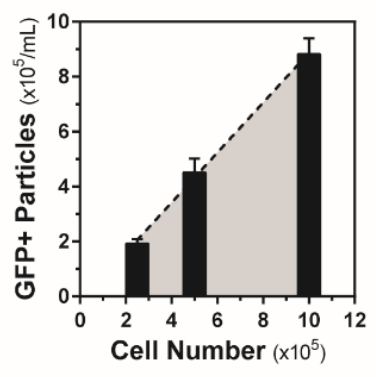

B)

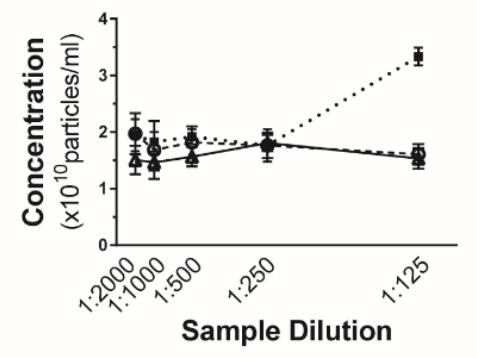

C)

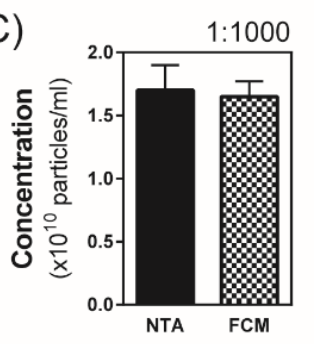

D)

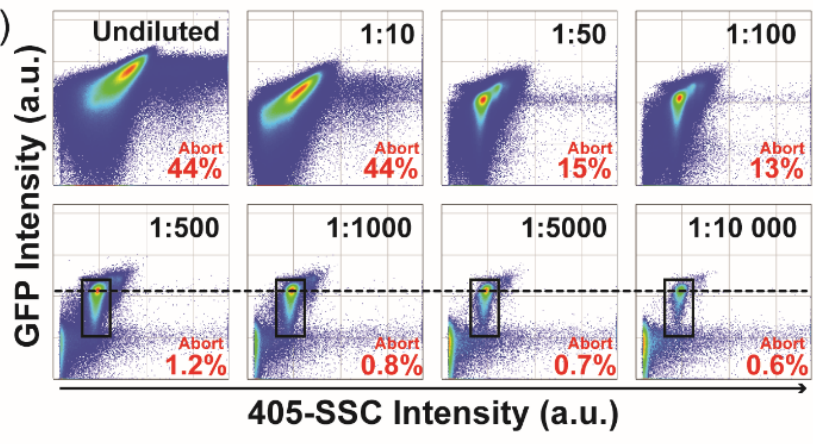

E)

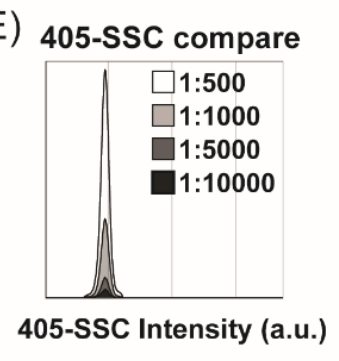

F)

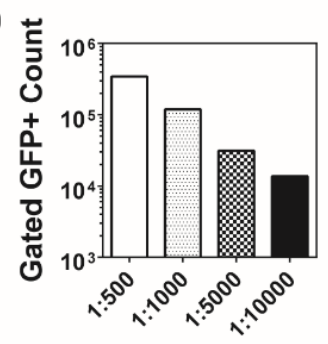

Supplementary Figure 1. Coincidence detection and determination of virus particle concentration through serial dilutions using FCM and NTA. A) Analysis of equivalent volumes of supernatants collected from an MLV infected cell line correlating seeding densities of cells to the amount of virus produced; $\mathrm{n}=6$. B) Calculated particle concentration of MLVsfGFP in undiluted supernatant based on concentrations measured by NTA in serial dilutions $(n=3)$. C) The calculated concentration of undiluted MLVsfGFP supernatant based on measurements determined by NTA and FCM using samples diluted 1:1000 (n=3). D) Flow cytometry analysis of MLV dilutions showing the abort rates and increase in measured GFP fluorescence intensity and 405-SSC intensity of MLVsfGFP at the highest concentrations. E) An overlay of the events from the highest dilutions to compare 405-SSC scatter intensities. F) Linear correlation of GFP+ events (gated in (Fig. 1A) with dilution factor. 
Tang et al., 2019
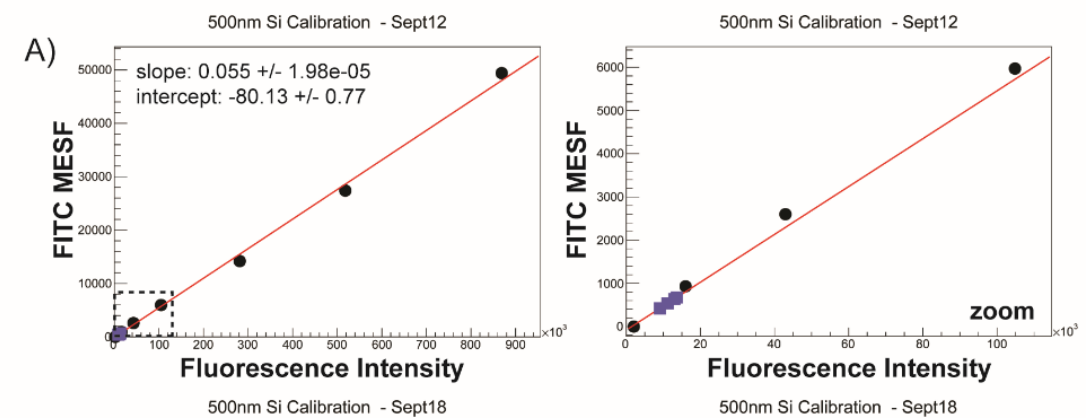

B)
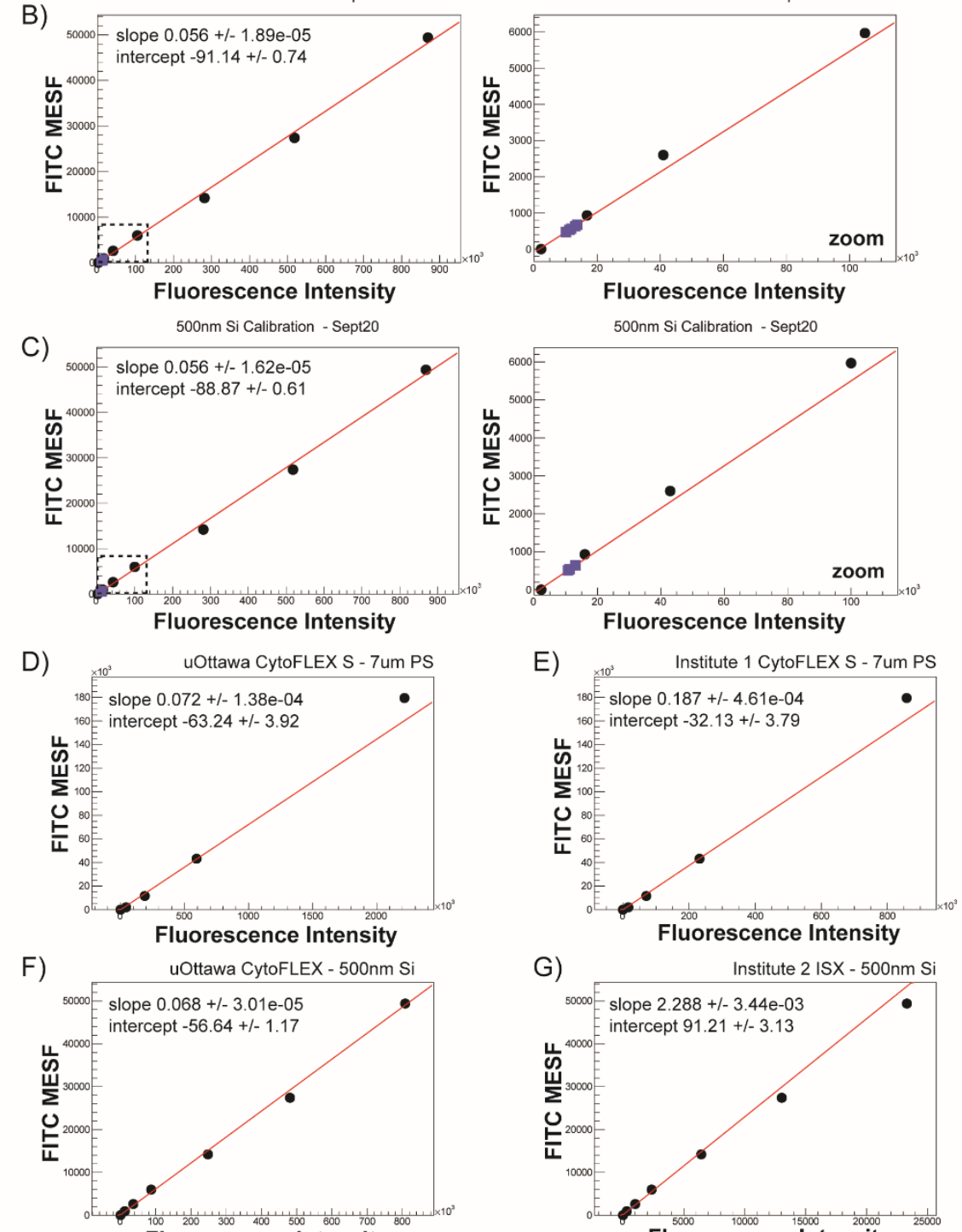

Fluorescence Intensity

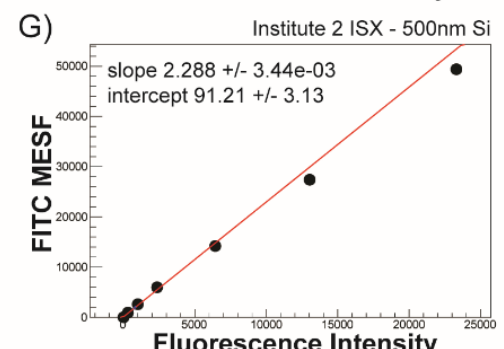

Supplementary Figure 2. Calibration Curves for MESF calculations for Figures 2 \& 3. (A to C) Calibration curves for 500nm Si MESF beads for data collected on 3 separate dates for MESF values summarized in Figure 2G. (D) Calibration curve for uOttawa CytoFLEX S using $7 \mu \mathrm{m}$ PS FITC MESF Beads. (E) Calibration curve for Institute 1 CytoFLEX S using $7 \mu \mathrm{m}$ PS FITC MESF Beads. (F) Calibration curve for uOttawa CytoFLEX S using $500 \mathrm{~nm}$ Si FITC MESF Beads. (G) Calibration curve from Institute 2 ISX using $500 \mathrm{~nm}$ Si FITC MESF Beads. 
Tang et al., 2019

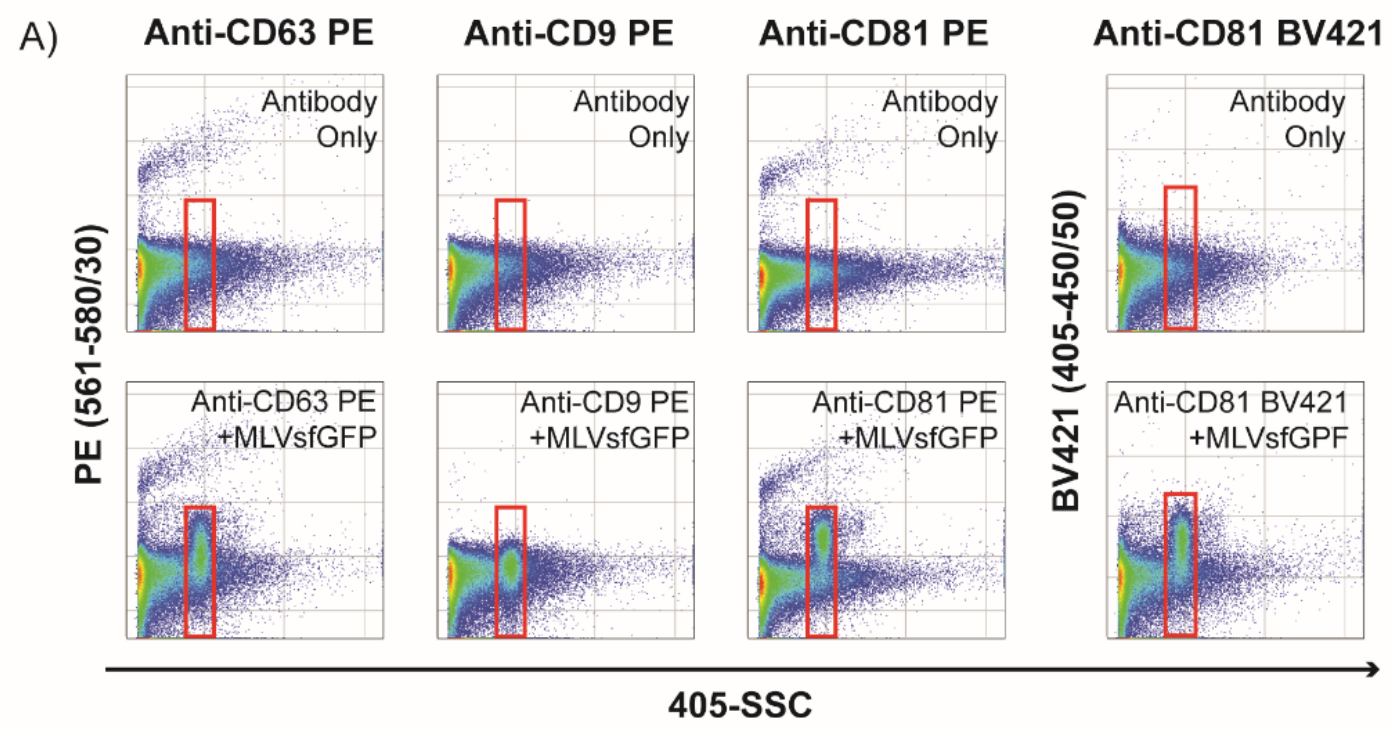

B)

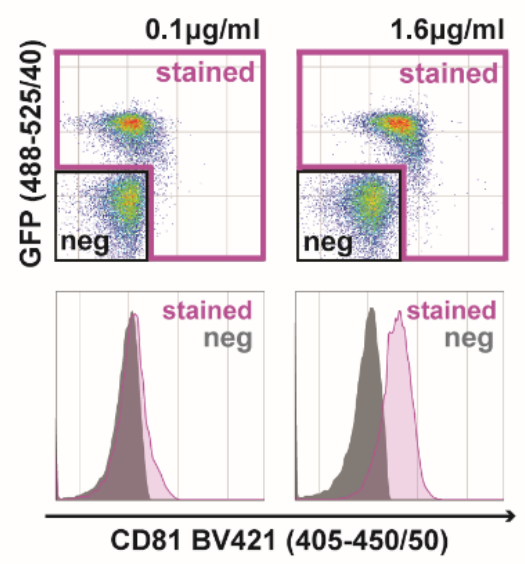

C)

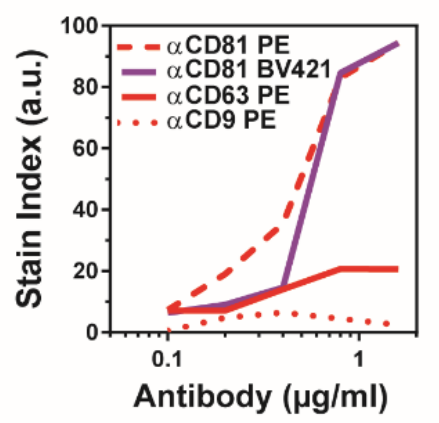

D) MLV infected cells

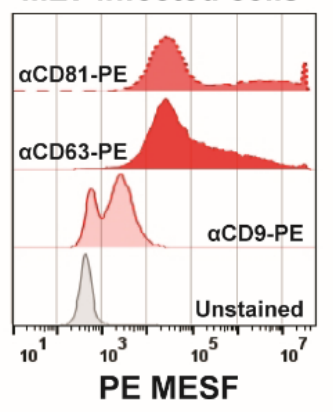

Supplementary Figure 3. Anti-tetraspanin labeling of MLVsfGFP and MLVsfGFP infected cells. (A) Gating strategy for virus events to remove antibody aggregates using antibody only controls. All events are displayed as PE Intensity vs. 405-SSC Intensity for anti-mouse PE conjugates for CD9, CD63, and CD81. (B) Gated events from (A) are displayed as GFP vs. PE Intensity. These events were then calibrated to be displayed as FITC MESF vs PE MESF in Figure 6. (B) Gating strategy used to identify stained and negative populations used to calculate SI. (C) SI for anti-CD81-PE, anti-CD81-BV421, anti-CD63-PE, and antiCD9-PE at concentrations from $0.1 \mu \mathrm{g} / \mathrm{ml}$ to $1.6 \mu \mathrm{g} / \mathrm{ml}$. D) Anti-tetraspanin labeling of chronically infected producer cells for MLVsfGFP, representative histogram of $n=3$. 
Tang et al., 2019
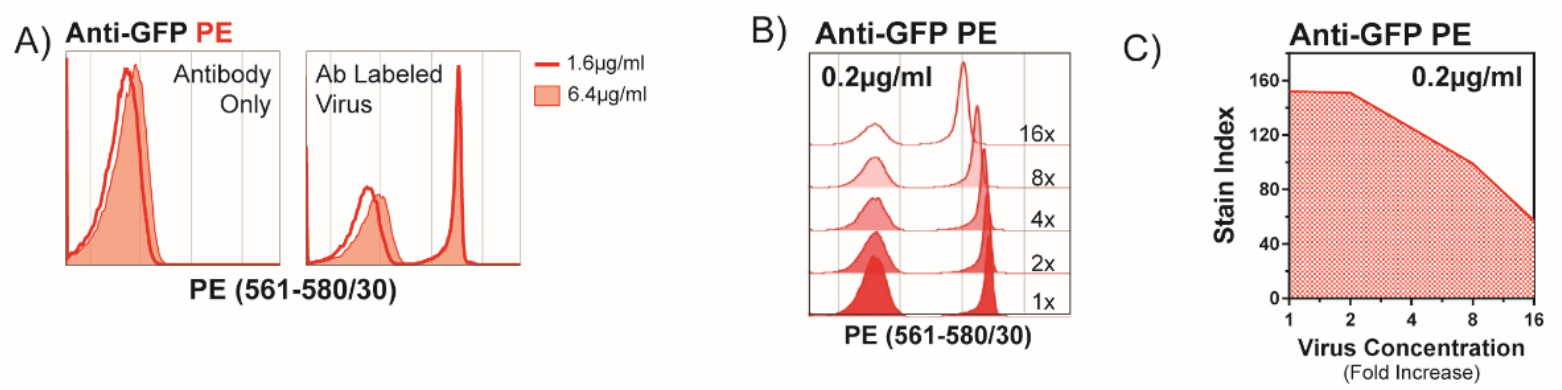

Supplementary Figure 4. Effect of changes in particle and antibody concentration on the stain index of antibody labeled MLVsfGFP. (A) Histogram overlay of MLVsfGFP labeled with anti-GFP-PE at increasing virus concentrations while maintaining staining concentration of $0.2 \mu \mathrm{g} / \mathrm{ml} .1 \mathrm{x}$ is the original virus concentration used to obtained optimal stain index at $0.2 \mu \mathrm{g} / \mathrm{ml}$. (B) Stain index calculated from (A). (C) Histogram overlay of anti-GFP-PE antibody-alone at $1.6 \mu \mathrm{g} / \mathrm{ml}$ and $6.4 \mu \mathrm{g} / \mathrm{ml}$ (left panel) and anti-GFP-PE labeled MLVsfGFP + MLVnoGFP (right panel) showing staining of the MLVsfGFP population is saturated at $1.6 \mu \mathrm{g} / \mathrm{ml}$. 
Tang et al., 2019

\section{A)}
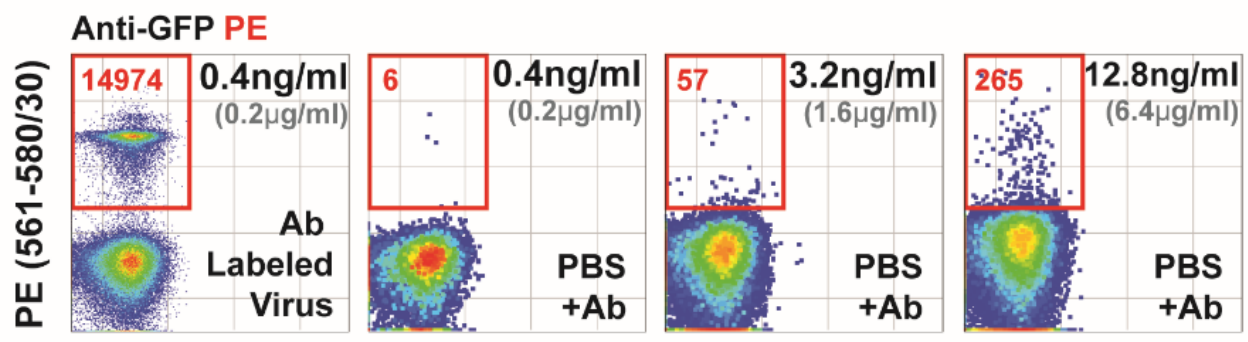

B)
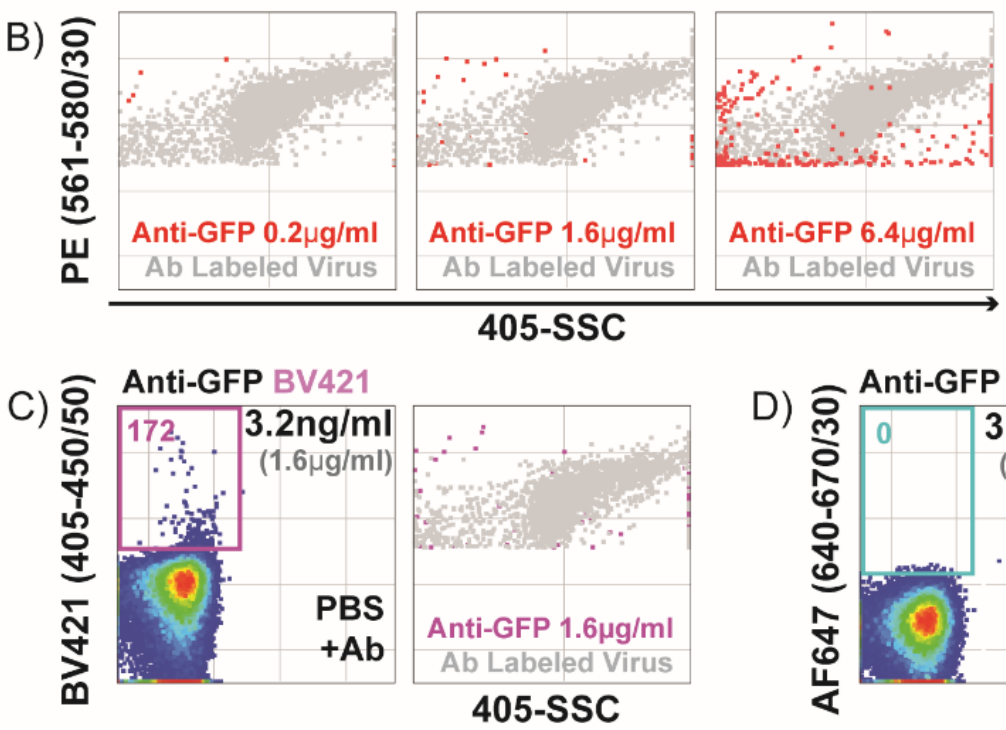

D)

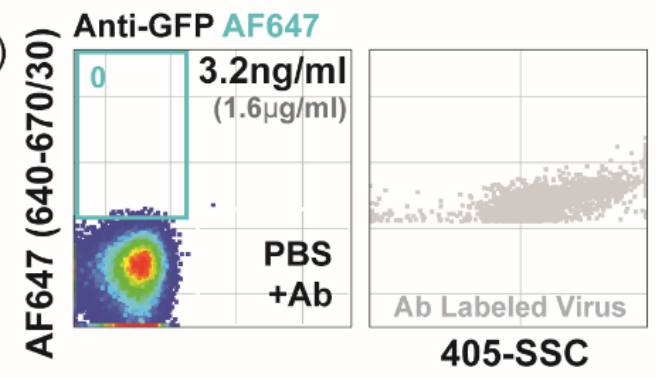

Supplementary Figure 5. Detection of antibody aggregates in anti-GFP conjugates. (A) Dilutions of antiGFP-PE antibody alone were analyzed at $0.2 \mu \mathrm{g} / \mathrm{ml}$ (optimal staining concentration), $1.6 \mu \mathrm{g} / \mathrm{ml}$ and $6.4 \mu \mathrm{g} / \mathrm{ml}$. The first panel on the left denotes MLVsfGFP stained at a concentration of $0.2 \mu \mathrm{g} / \mathrm{ml}$. Concentrations in black indicate the actual concentration of antibody as it is diluted for analysis on the cytometer. Values in red are PE+ event counts within the red gate. (B-D) Overlays of MLVsfGFP labeled at optimal staining concentration (gray events) with increasing concentrations of (B) anti-GFP-PE; (C) $1.6 \mu \mathrm{g} / \mathrm{ml}$ of anti-GFP-BV421; and (D) anti-GFP-AF647. Representative plots for three independent experiments are shown. 